\title{
Flow and noise from septa nozzles
}

\author{
K.B.M.Q. Zaman ${ }^{1}$ and J.E. Bridges ${ }^{2}$ \\ NASA Glenn Research Center \\ Cleveland, $\mathrm{OH} 44135$
}

\begin{abstract}
Flow and noise fields are explored for the concept of distributed propulsion. A model-scale experiment is performed with an 8:1 aspect ratio rectangular nozzle that is divided into six passages by five septa. The septa geometries are created by placing plastic inserts within the nozzle. It is found that the noise radiation from the septa nozzle can be significantly lower than that from the baseline rectangular nozzle. The reduction of noise is inferred to be due to the introduction of streamwise vortices in the flow. The streamwise vortices are produced by secondary flow within each passage. Thus, the geometry of the internal passages of the septa nozzle can have a large influence. The flow evolution is profoundly affected by slight changes in the geometry. These conclusions are reached by mostly experimental results of the flowfield aided by brief numerical simulations.
\end{abstract}

\section{Introduction}

This model-scale investigation addresses basic features of flow and noise from a distributed propulsion system [1, 2]. In such a system the exhausts of multiple propulsors are gathered into a common stream before exiting the nozzle. The concept, considered for some modern aircraft designs, affords ease of integration of the propulsion system with the airframe. In one version of the concept each propulsor duct is driven by an electric fan, the power being generated by engines suitably mounted on the airframe. With such a 'hybrid' system the need for heavy power trains is eliminated and system studies do indicate a net benefit in efficiency. From an aeroacoustics point of view, however, there are concerns. An immediate question is whether the noise from the multiple jets would be equal to or greater than that from an equivalent single jet. When multiple jets are placed side by side sometimes they resonate and produce more noise. In order to address these concerns an experimental study has been carried out at NASA Glenn Research Center (GRC).

Preliminary results of this study were presented in [3]. One of the key findings was that the noise from the septa nozzle could actually be less than that from the equivalent single nozzle. Continued study made it apparent that the flow as well as the radiated noise could be quite sensitive to the geometry of the passages of the individual propulsion ducts. Specifically, it was shown in [3] that two septa nozzles of identical design produced very different flow fields apparently due to slight irregularities in the fabrication. Instead of remaining symmetric the cellular flow

\footnotetext{
${ }^{1}$ Inlets \& Nozzles Branch, Propulsion Division.

${ }^{2}$ Acoustics Branch, Propulsion Division.
} 
structure from one nozzle went through a pairing process downstream whereas no such pairing occurred with another. An objective of the continued study was to investigate this anomalous behavior and obtain a clearer understanding of the impact of variations in the geometry of the propulsion ducts on the flow and noise. Key results from the entire investigation are summarized in this paper.

\section{Experimental Facility and Procedure}

An open jet facility (referred to as 'CW17') at NASA GRC is used for the experiment. Compressed air passes through a 30" diameter plenum chamber before exhausting through the nozzle into the ambient of the test chamber. Only cold (unheated) flows are considered and further description of the facility can be found in earlier publications [3,4]. An 8:1 aspect ratio nozzle, referred to as 'NA8Z' [3], is used in the current experiment. The nozzle exit has dimensions of 5.339"x0.658" and thus an equivalent diameter, $D=2.12$ ". A picture of the facility is shown in Fig. 1(a). Figure 1(b) shows another view of the nozzle with a single hot-wire probe in the foreground. Detailed hot-wire surveys at a low Mach number as well as Pitot probe surveys at high Mach numbers are conducted.

The septa geometry is created by placing an insert into the nozzle. The insert fits snugly into the nozzle and is held in place under flow because of the convergent geometry of the nozzle. All data presented in this paper are obtained with inserts having six passages (or ducts) and five septa. These were fabricated by additive manufacturing process (3-D printing). Figure 1(c) shows pictures of two inserts having different internal passage geometries. The view is from the upstream end. For the one on the top, each passage starts with an almost square entrance, transitions to a circular cross-section and then transitions back to a rectangular exit. The circular cross-section would be the location of the electric fan in the hybrid propulsion system. For the one on the bottom, the passage is rectangular throughout; this creates a baseline geometry without changes in cross-sections of the passage at its entrance and exit. These two inserts are referred to as the 'Fsh3' and the '6Rec' cases, respectively; the notations are further discussed in the following. In both cases the flow continually converges throughout the passage. As reported in [3], various other septa geometries (all with rectangular-circular-rectangular passages, as with the Fsh3 case at the top of Fig. 1c) were tried in an effort to find maximum noise reduction. Four of those are shown by the schematics in Fig. 1(d). These will be examined for flow-field and noise details. For these cases, only the end geometry of the septa separating the passages is varied. The 'Fshx' case has the septa ending flush with the exit. Over time, several 3-D prints of the 'Fshx' case were used in the experiment; data from two of them, the original one referred to as 'Fsh0' and a later one referred to as 'Fsh3', will be discussed in this paper. The 'Insd' case has the septa stopping 0.25 " inside from the exit. The 'OutF' case has septa protruding out with full width by 0.175 ". The septa in the 'Sclp' case are scalloped at the end (semi-circular cut-out). The geometries are depicted in the side views in Fig. 
1(d). Data from all of these cases are compared with that from the baseline case without any insert (denoted as 'Bsln').

The test cell has acoustic linings on the ceiling and upper walls, and with proper preparation, qualitative noise measurements are possible. For the present study, comparative spectral levels from geometry to geometry were examined and the facility was considered adequate for that purpose. Microphones $(1 / 4 ", \mathrm{~B} \& \mathrm{~K}$ 4135 ) held fixed on an overhead arm were used to obtain the noise data. The microphone polar location $\theta$ was referenced with respect to the jet's downstream axis. Spectral analysis was done over 0-50kHz with a bandwidth of $50 \mathrm{~Hz}$. All spectral data in this paper pertain to a microphone location on the minor axis plane, that is, on the broad side of the nozzle. A single hot-wire was used for flowfield surveys at low jet Mach numbers $\left(M_{j}\right)$. Limited data at low $M_{j}$ were also obtained with X-wire probes. A rake of three Pitot probes was used for flowfield surveys at high $M_{j}$. All dimensions quoted in the paper are in inches. Further details of the measurements are discussed along with the relevant results.

Flow simulations were made along with the design iterations during the investigation. Reynolds-Averaged Navier-Stokes simulations were made using the Mentor Graphics flow simulation package [5] sold by Dassault Systemes with their SolidWorks ${ }^{\mathrm{TM}}$ computer-aided-design (CAD) software. This industrial-grade flow solver uses immersed boundary meshing and a relatively simple $k$ - $\varepsilon$ turbulence model to readily produce a credible flow solution using a desktop computer for most subsonic problems. The nozzle flow lines were created in the SolidWorks ${ }^{\mathrm{TM}} \mathrm{CAD}$ package and the flow simulation results were used in screening nozzle flow concepts and confirming overall flow features observed in the experiment.

\section{Results}

Figure 2 compares sound pressure level (SPL) spectra at two jet Mach numbers $\left(M_{j}=1\right.$ and 0.9$)$. The data are for $\theta=90^{\circ}$ on the broad side of the jet. Comparison between the flush (Fsh3) and the baseline (Bsln) cases is made in Fig. 2(a). There is a significant reduction of the spectral amplitudes on the low frequency end. With the insert, there is flow blockage; the equivalent diameter is smaller by about $11 \%$. It can be estimated that the reduction in spectral amplitudes due to the blockage should be about $1.3 \mathrm{~dB}$. The amplitudes on the low frequency end have reduced in excess of $5 \mathrm{~dB}$ and thus cannot be due only to area blockage. SPL spectra for the case with rectangular passages throughout (6Rec) are shown in Fig. 2(b). Here, the comparison is made between the 6Rec case and the Fsh3 case (the two inserts of Fig. 1c). Clearly, the case with rectangular-circular-rectangular passage (Fsh3) yielded more noise reduction. A comparison with the data in Fig. 2(a) makes it clear that the 6Rec case also reduced noise relative to the Bsln case but not as much as seen with the Fsh3 case. 
Similar comparisons of the SPL spectra for the other cases of Fig. 1(d), are shown in Figs. 3(a)-(d). Again, in each figure the data for an individual case is compared with the Fsh3 data. It is apparent that with both the Insd and the OutF cases (Figs. 3a and b) the noise reduction is not as much as with the Fsh3 case. The Sclp case in Fig. 3(c) is seen to be as effective as the Fsh3 case. Many other designs were tried in [3] and the Fsh3 (or Fsh0) case produced the most noise reduction. Recall that the Fsh3 and Fsh0 cases are the 3-D prints of the same design; as it will be shown shortly, the two produced quite different flow fields downstream. In spite of the flowfield differences these two produced comparable noise results (Fig. 3d). Close scrutiny suggests that the Fsh0 case may have yielded slightly better noise reduction.

The flowfield characteristics for these nozzles are explored next. Figure 4 shows hot-wire survey data at $x / D=2$; as stated in $\S 2, D$ is the equivalent diameter of the baseline nozzle. Contours of mean velocity (normalized by jet velocity at the nozzle exit) on the cross-sectional plane are plotted for a low Mach number $\left(M_{j}=0.265\right)$. The data are for different septa designs as indicated in the figure caption. For all cases the flow started as six high velocity cells just downstream of the nozzle exit (see further data in the following). For the Fsh3 case in (a), the six cells have transformed into five high velocity 'blobs' by $x / D=2$. This behavior is understandable. There are five septa for the six passages and apparently pairs of streamwise vortices form at the ends of each septum. The vortex pairs pull the flow outward causing high velocity flow from adjacent cells to congregate in front of each partition, causing the formation of the five high velocity blobs.

Figure 4(b) shows the corresponding velocity distribution for the Fsh0 case. As stated before, this insert was of the same design as the Fsh3 case. However, the flowfield is quite different from that seen in Fig. 4(a). By the measurement station at $x / D=2$ the six original high velocity cells have gone through a pairing process to yield three cells. An asymmetry has also developed. Note that the middle cell is not centered at $z=0$. The Fsh 0 case was the first septa insert tried in this investigation; since then several more inserts of the same design were printed and most showed a behavior as seen in Fig. 4(a). The peculiar behavior with the Fsh0 case is due to small differences in the internal geometries of the septa passages. The 3-D printing process was not precise; the tolerance was approximately $\pm 0.010 "$. Small differences in the dimensions from septum to septum were possible even though nothing was visually obvious. The flowfield difference between Figs. 4(a) and (b) is the anomalous behavior noted in $\S 1$. This is pursued further in the following.

Figure 4(c) shows flowfield data for the 6Rec case, at $x / D=2$ and $M_{j}=0.265$. There is no pairing activity. Neither is there a transition to five high velocity blobs as seen in Fig. 4(a). The six high velocity cells are still discernible at the measurement station. Recall that this case has rectangular cross-section throughout each passage, as opposed to the rectangular-circular-rectangular geometry with the Fsh3 case. The difference in the internal passage geometry made 
a significant difference in the flowfield as well as in noise (Fig. 2). The three other cases in Fig. 4 with various shapes of the septa ends, all with rectangular-circular-rectangular internal passage, exhibit similar patterns as seen for the Fsh3 case in Fig. 4(a). Note that the Sclp case (Fig. 4f) produced almost identical pattern as with the Fsh3 case, and these two also have practically identical noise signatures (Fig. 3c). The high velocity blobs are somewhat diffused for the Insd and OutF cases (Figs. $4 \mathrm{~d}$ and 4e). The noise reductions with the latter two cases were not as much as with the Fsh3 case (Figs. 3a and 3b).

Corresponding flowfield data at a high Mach number $\left(M_{j}=0.90\right)$ for selected cases are shown in Figs. 5. These data at $x / D=2$ were obtained by Pitot probe surveys. The patterns for the Fsh3, Fsh0 and 6Rec cases in Figs. 5(a), (b) and (c) are quite similar to the patterns seen at low speeds in Figs. 4(a), (b) and (c), respectively. These flow fields are a result of streamwise vortex interactions and those interactions are obviously not affected significantly by compressibility effects.

Figures 6, 7 and 8 document the streamwise evolution of the flow for the Fsh3, Fsh0 and 6Rec cases, respectively. These hot-wire data at $M_{j}=0.265$ are shown for five streamwise locations, as indicated. In both Figs. 6 and 7 , the migration of the flow to form high velocity blobs downstream of each septum can be noted. However, for the Fsh0 case (Fig. 7) there is further interaction with increasing downstream distance. Counting from the top, high-velocity blobs 1 and 2, and 3 and 4 merge, while blob 5 at the bottom is left alone. This is how the asymmetry in the flow develops. In contrast, the five blobs for the Fsh3 case (Fig. 6) continue to retain their identity until being diffused by $x / D=4$. For the 6 Rec case in Fig. 8 , on the other hand, there is neither an inter-cell migration nor a pairing activity farther downstream. The six high velocity cells retain their identity until getting diffused by $x / D=4$. It is apparent that streamwise vortex pairs are formed at the ends of the septa for the rectangular-circular-rectangular passage geometry (Fsh3 and Fsh0); (limited X-wire data for the Fsh3 case are presented shortly). Such vortices are absent in the $6 \mathrm{Rec}$ case with the rectangular passage. It is also apparent that some sort of nonuniformity is introduced in the vortices with the Fsh0 case that lead to the subsequent pairing activity.

As already stated, the Fsh0 and the Fsh 3 cases were fabricated from the same CAD files and were supposed to be identical. Obviously small variations in the septa geometry due to the uncertainty in the fabrication process resulted in the observed difference in the flow. The variations could not be discerned visually or through cursory measurements with calipers. The two specimens were then inspected by optical methods (by Inspection Engineering, Westlake, $\mathrm{OH}$, using an apparatus called 'Flash Smartscope'). Dimensions deemed critical are listed in Table 1. The sketch on the side of the table provides definitions of the parameters; $S 1$ and $S 2$ are the widths of a passage at the inlet and outlet, respectively, and $d$ is the diameter of the circular cross-section in the middle. It can 
be seen that up to 0.024" differences in the dimensions occurred in certain parameters between the two inserts (see, the values of $S 1$ for passage \#3 in Table 1).

Thus, the maximum difference was about $2.8 \%$ of the design width and no other gross anomalies in the dimensions stood out. The measurements pertained to the central plane of each passage and there were variations in the transverse direction. However, from visual inspection variations worse than the observed maximum were unlikely. In any case, these data were not helpful in pinpointing the cause for the difference in the flowfield. At this point, several new inserts were fabricated by deliberately varying the dimensions of the passages. Specifically, the dimensions $S 2$ and $d$ were varied from passage to passage in a systematic manner. The notion was that such variations would change the contraction ratio from the circular to the rectangular exit section and thereby affect the strength of the secondary flow that in turn would affect the strength of the streamwise vortices. Flowfield surveys for four such design cases are shown in Fig. 9. For each case data at $x / D=2$ and 4 are shown with the design change indicated by the sketch on the left. The changes are as follows. The blue circles indicate that the diameter $d$ was decreased by 0.025 " from the nominal value of $0.855^{\prime \prime}$ while the red circles indicate an increase in $d$ by 0.025 ". Similarly, the blue rectangles indicate a decrease in the outlet width $S 2$ by 0.025 " and the red rectangles an increase in $S 2$ by $0.025 "$. Noting that the 3-D printing process is not precise the exact dimension changes are not guaranteed. However, the changes were large enough to affect the overall contraction ratios in the desired directions.

From Fig. 9 it is apparent that any of the deviations from the standard design may affect the flowfield evolution in some manner. This is evident from the nonuniformity in the spacing of the high-speed blobs at $x / D=2$, although the effect was only slight and nowhere near as strong as seen with the Fsh0 case. Many other combinations were tried. The closest resemblance with the Fsh0 case was achieved by the design in Fig. 10. Here, both $d$ and $S 2$ were varied to accentuate the effect. A tendency for pairing as with the Fsh0 case can be seen by $x / D=2$. By $x / D=4$ the flow became quite similar to that seen with the Fsh0 case (Fig. 7).

Limited Reynolds-Averaged Navier-Stokes flow simulations were conducted during the exploration of geometric impacts. Streamwise evolution of mean velocity data for the Dsn9 case (Fig. 10) is shown in Fig. 11. A very similar flowfield evolution is noted. Corresponding CFD results for the Fsh3 case are shown in Fig. 12. When compared with the data in Fig.3, again a very similar evolution can be seen. These CFD results confirm that indeed a small variation in the internal passage dimensions can impact the flowfield quite significantly leading to the flow patterns observed in the experiment.

Finally, X-wire hot-wire measurements were performed for the Fsh3 case in order to explore further details of the flow field. Two X-wires, one in $u$-v and another in $u$ - $w$ configuration, were traversed in $z$ through the same 
measurement grid points. The two probes were separated by $0.96 "$ and the two sets of data were shifted during postprocessing to coincide with each other, providing time-averaged values for all three components of mean velocity and normal stresses at each point. In addition, the Reynolds stresses $u v$ and $u w$ were also obtained. Unfortunately, the spatial resolution was not good for resolving the fine details of the flow containing a multitude of vortices. The measurements were carried out only at $x / D=2$ in an effort especially to identify the streamwise vortices. Note that the $\mathrm{X}$-wire probes had to be inserted straight into the flow with probe support parallel to the $x$-direction; (the single hot-wire was inserted at an angle and traversed in $y$-direction in order to minimize probe interference). Thus, only a little over half of the flowfield was covered in the X-wire measurements since interference from the probe support would be large when reaching the far end in negative $z$. A few of the flow properties are shown in Fig. 13. The mean velocity $U$ can be compared with the single hot-wire data in Fig. 6. For normal stresses, only $u$ ' and $v$ ' are shown while $w^{\prime}$ ' was noted to be very similar to $v^{\prime}$ in amplitude and distribution. Peak $u$ '-amplitude (0.163) is found to be much larger than peak $v$ '-amplitude (0.106) or peak $w$ '-amplitude (0.102). The two Reynolds stress data are also shown. Finally, the streamwise vorticity data are shown at the bottom right corner of Fig. 13. Even though not orderly and crisp, the $\omega_{x}$ data leave no doubt that pairs of counter rotating vortices occur on either side of the flow field. Recall that the high speed blobs in the $U$-distribution occur in front of each of the five septa. A comparison of the $\omega_{x}$ data with the $U$ data show that indeed vortex pairs of opposite sense occur at the ends of each septum.

\section{Concluding Remarks}

A model-scale experiment is conducted with an 8:1 aspect ratio rectangular nozzle that is divided into six passages by five septa. Plastic inserts placed inside the nozzle create the septa geometry. The inserts are fabricated by additive manufacturing process (3-D printing). It is found that the noise radiation from the septa geometry can be significantly lower than that from the baseline rectangular jet. Up to $5 \mathrm{~dB}$ noise reduction is noted on the low frequency end of the spectrum. The septa inserts cause a flow blockage amounting to about $11 \%$ reduction in the equivalent diameter of the nozzle. Rough estimates show that this should correspond to about $1.3 \mathrm{~dB}$ reduction in noise; thus, the observed noise reduction is not due only to flow blockage.

The noise reduction occurs with the septa design where the flow passage goes through a rectangular-tocircular-to-rectangular transition. It is observed that when the passage is rectangular throughout there is also some noise reduction that appears to be mostly due to the flow blockage. Flowfield surveys suggest that pairs of streamwise vortices are introduced with the rectangular-to-circular-to-rectangular passages. These pairs occur at the ends of each of the five septa that separate the six passages. The vortex pairs pull the flow outward and as a result the six high velocity cells originally issuing from the nozzle transform into five high velocity blobs shortly downstream. In contrast, with the rectangular-throughout passage case the six cells 
retain their identity far from the nozzle exit until getting diffused due to turbulence. This suggests that there are no significant streamwise vortices in the latter case. From these considerations it may be inferred that the introduction of the streamwise vortices in the former case is at the root of the noise reduction.

The introduction of the streamwise vortices and the resultant evolution of the flowfield are found to be sensitive to upstream passage geometry. In fact, the experiments discussed in this paper were prompted by an 'anomalous' behavior noted earlier in the study. Two septa inserts fabricated with the exact same design produced very different flow fields. In one, the six high velocity cells produced five regions of high velocity flow, as discussed in the previous paragraph. In another, the five regions went through a further pairing process that produced an asymmetric flowfield with three high-velocity regions. It is inferred that this difference in behavior resulted from slight imperfections in the geometries of the latter insert due to inherent inaccuracies in the 3-D printing process. This was pursued by deliberately introducing nonuniformities in the dimensions of adjacent septa. Eventually with certain changes in the dimensions a similar 'anomalous' flowfield could be reproduced. Limited numerical simulation with the latter design confirmed the observed development of the flow field.

While most of the data presented in the paper pertain to a low jet Mach number it was found that the flow pattern observed with a given insert was the same at a high subsonic Mach number. Thus, the distribution and interaction of the streamwise vortices affecting the flow evolution must be largely independent of compressibility effects.

Therefore, the rectangular-to-circular-to-rectangular passage within a flow duct, necessary in electric propulsion (the fan has to be located at a circular cross section), has an added benefit of producing some noise reduction. The noise reduction stems from the introduction of streamwise vortices, which are apparently produced by secondary flow in the transitional passages. The key lesson of this work is that the strength and distribution of those vortices can be sensitive to the exact geometry of the passages. This should be borne in mind in the design of a distributed propulsion system and in fact may be taken advantage of for tailoring the flowfield downstream as well as for achieving some noise reduction.

\section{Acknowledgement:}

The work was supported by the Advanced Air Transport Technology (AATT) and Commercial Supersonic Technology (CST) Projects of NASA's Advanced Air Vehicle Program. Thanks are due to Dr. Cliff Brown for input in the initial planning of this experiment. Dr. Amy Fagan's help with data acquisition throughout the course of this investigation is also gratefully acknowledged. 


\section{References:}

[1] FELDER, J. L., KIM, H. D. AND BROWN, G. V., 2009, “Turboelectric Distributed Propulsion Engine Cycle Analysis for Hybrid-Wing-Body Aircraft", AIAA Paper 2009-1132, 47th AIAA Aerospace Sciences Meeting, Orlando, FL, 5 - 8 January.

[2] BERTON, J. J. AND HALLER, W. J., 2014, “A Noise and Emissions Assessment of the N3-X Transport”, AIAA Paper 2014-0594, AIAA SciTech Conf., , National Harbor, MD, 13-17 January. [3] ZAMAN, K.B.M.Q., BRIDGES, J.E., FAGAN, A.F. AND BROWN, C.A., 2016, “An Experimental Investigation of Jet Noise from Septa Nozzles", 22 $2^{\text {nd }}$ AIAA/CEAS Aeroacoustics Conference, Lyon, France, May 29-June 3.

[4] ZAMAN, K.B.M.Q., FAGAN, A.F., BRIDGES, J.E. AND BROWN, C.A., 2015, “An Experimental Investigation of Resonant Interaction of a Rectangular Jet with a Flat Plate”, J. Fluid Mech., vol. 779, pp. 751775. doi:10.1017/jfm.2015.453.

[5] SOBACHKIN, A., and G. DUMNOV. "Numerical Basis of CAD-Embedded CFD." Feb., 2014, [online]: https://www.solidworks.com/sw/docs/Flow_Basis_of_CAD_Embedded_CFD_Whitepaper.pdf

Table 1 Passage dimensions (inches) for Fsh0 and Fsh3 inserts. The widths $S 1, S 2$ and the circle diameter $d$ are defined in the sketch on right.

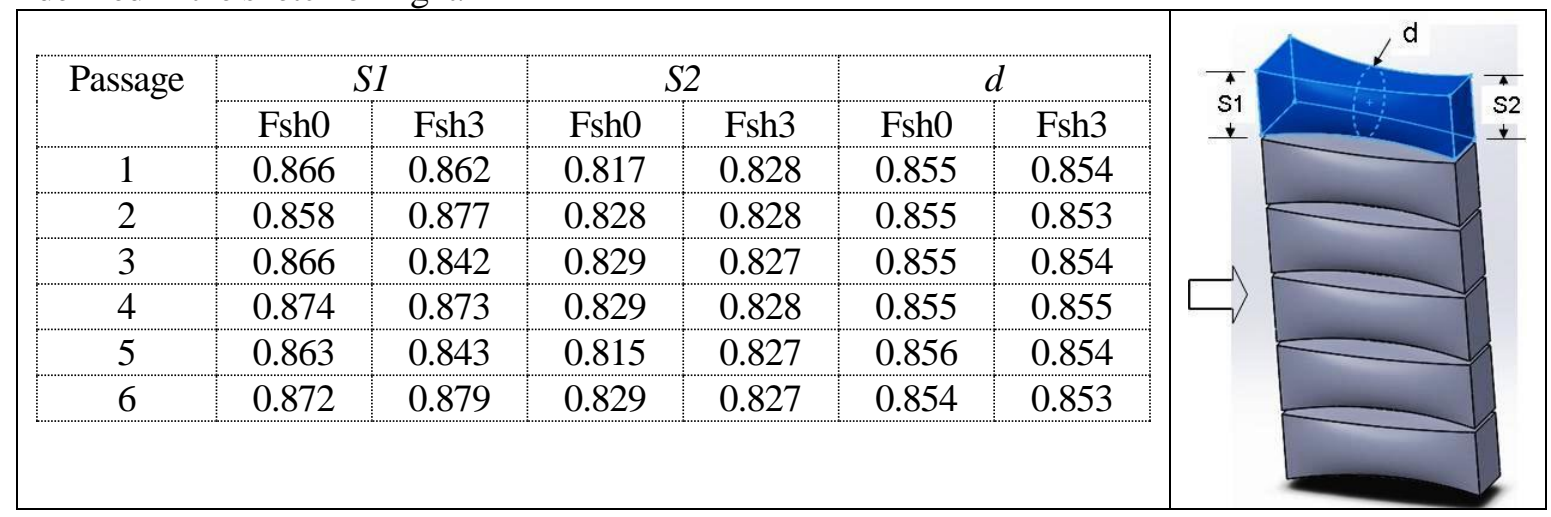
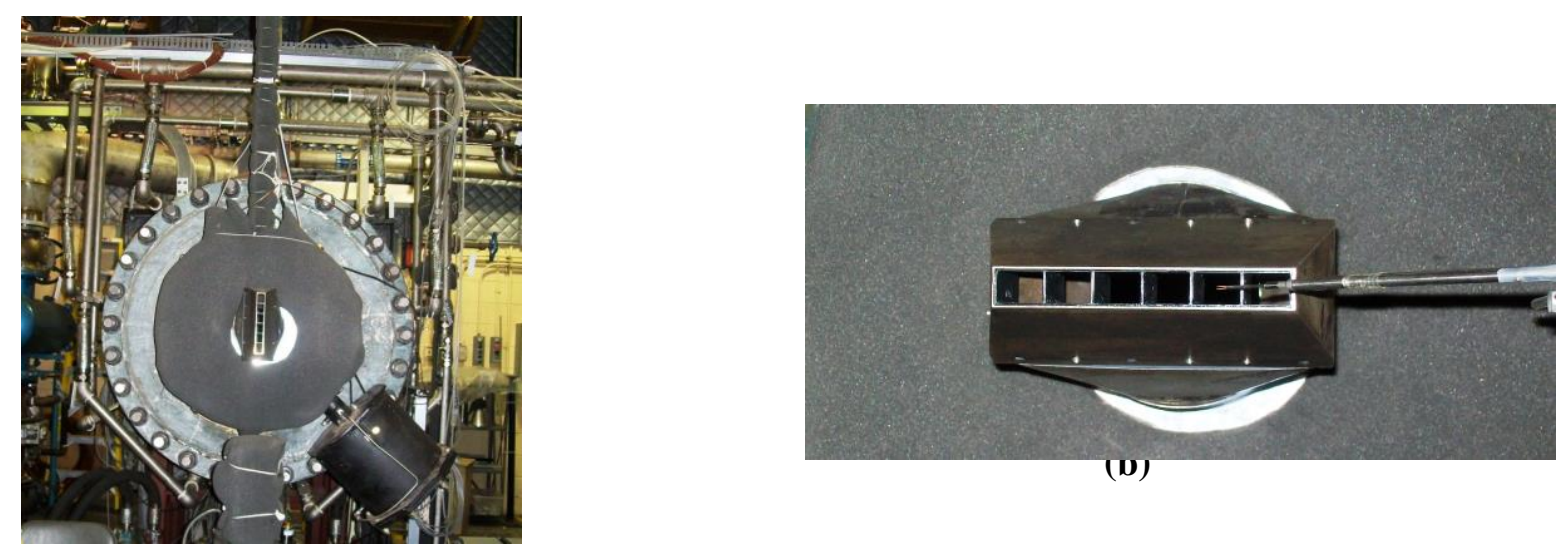
(a)
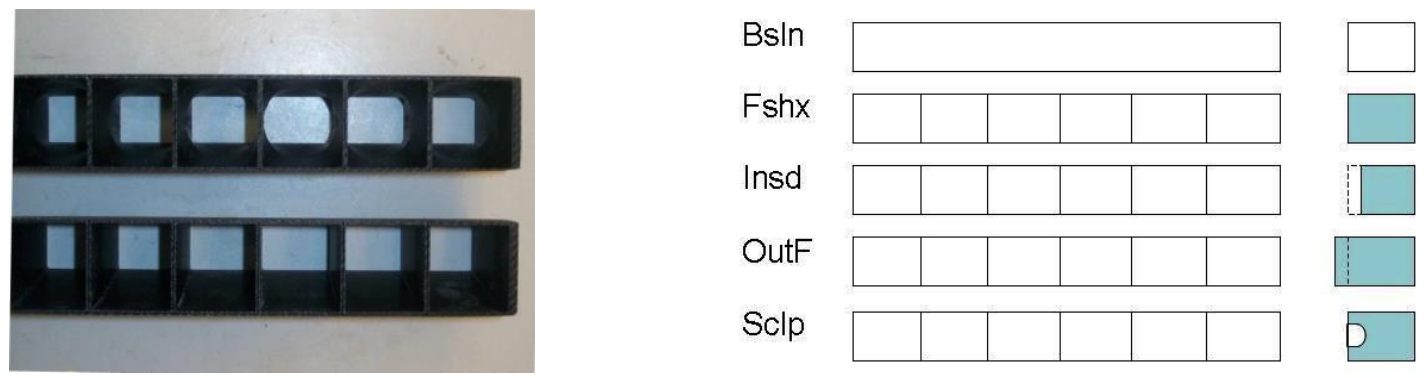

(d)

Fig. 1 Experimental setup. (a) Jet facility with 8:1 aspect ratio nozzle, (b) view of nozzle with hot-wire probe, (c) 6-passage insert with two different internal designs (top: rectangular-circular-rectangular passage, bottom: rectangular throughout passage), (d) schematic of exit shapes for different septa inserts.

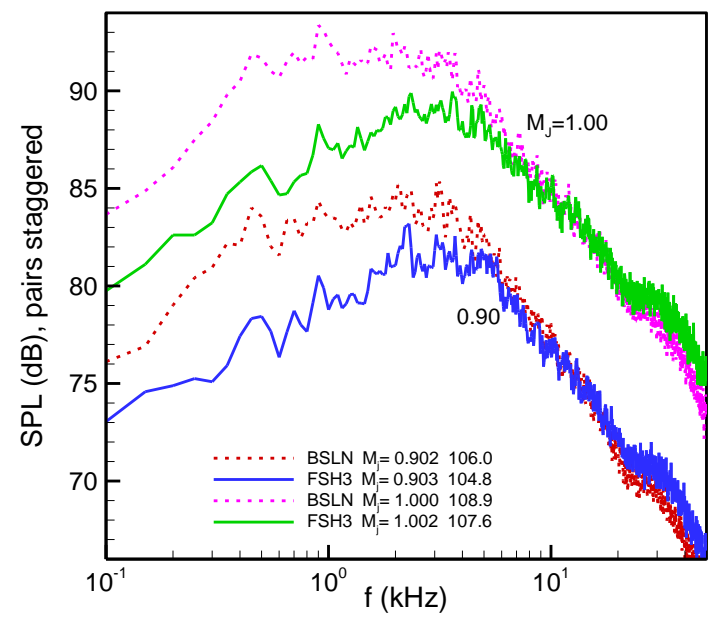

(a)

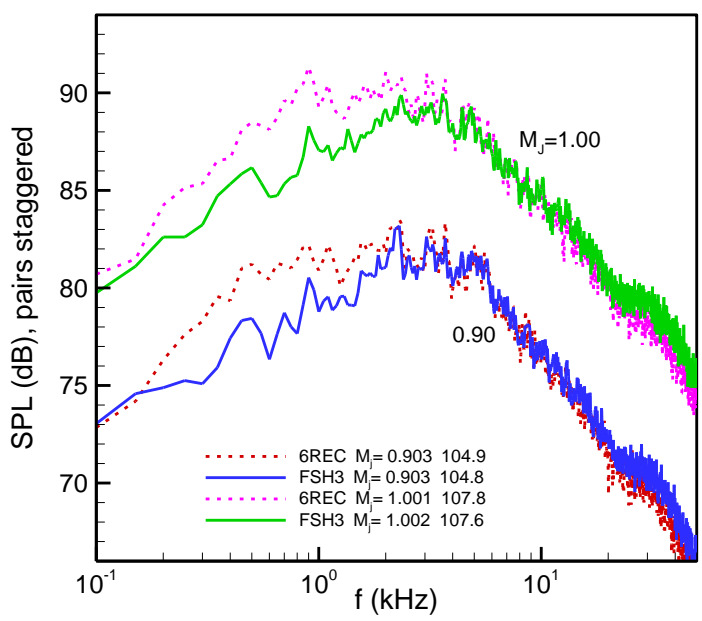

(b)

Fig. 2 Comparison of SPL spectra. (a) Flush case (Fsh3: solid lines) versus baseline case (Bsln, dotted lines); (b) Flush case (Fsh3, solid lines) versus case with rectangular passage throughout (6Rec, dotted lines). Data shown for two Mach numbers $\left(M_{j}\right)$ as indicated; microphone located on nozzle's minor axis plane at $\theta=90^{\circ}$. 


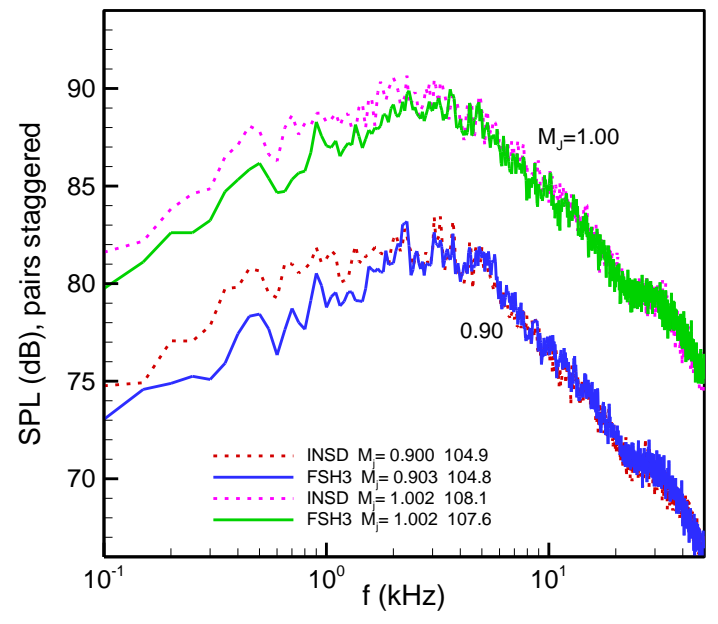

(a)

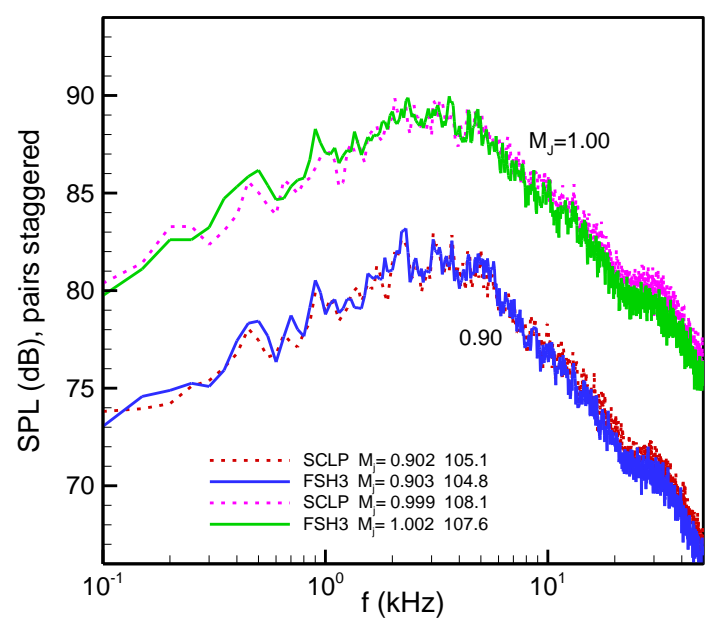

(c)

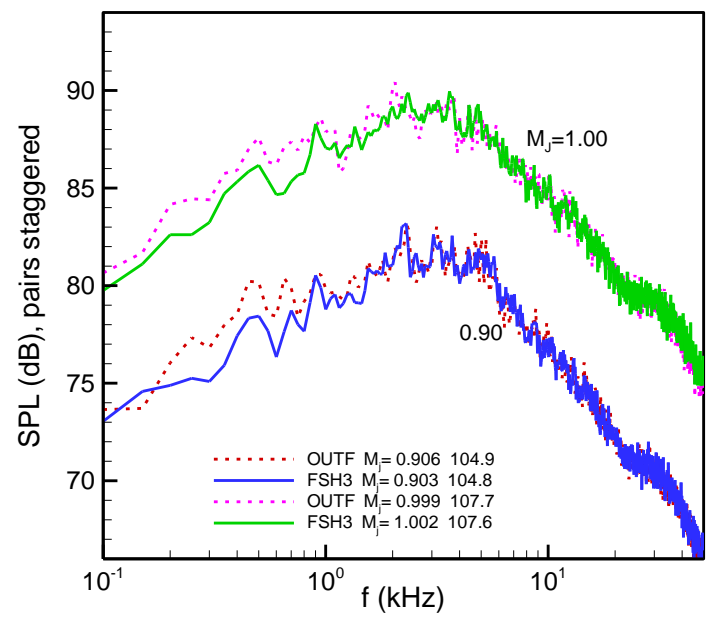

(b)

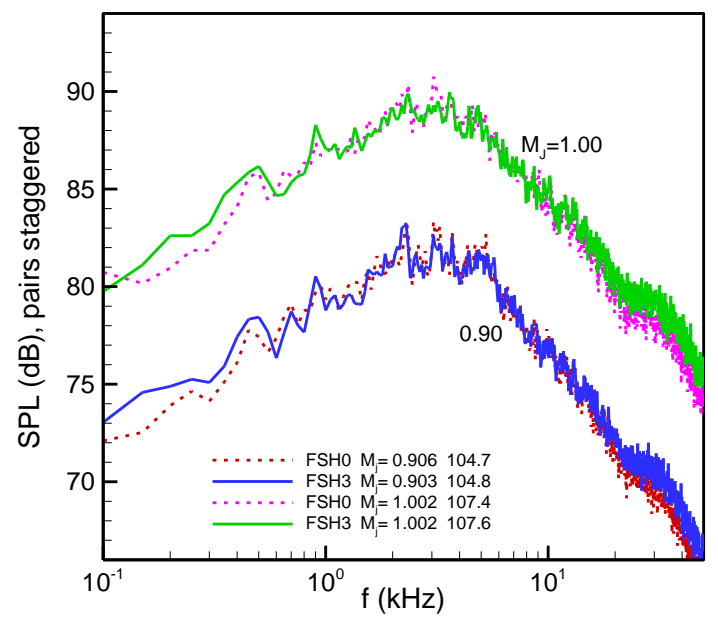

(d)

Fig. 3 Comparison of SPL spectra for various inserts (dotted lines) with data for the Fsh3 case (solid lines). (a) Insd; (b) OutF; (c) Sclp; (d) original flush case (Fsh0). In each figure data shown for two $M_{j}$; $\theta=90^{\circ}$.

(a)

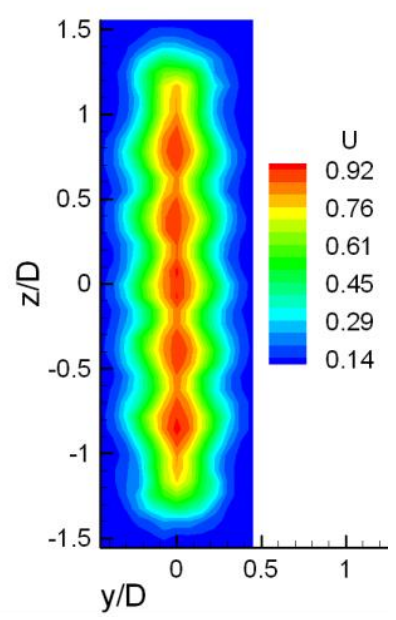

(b)

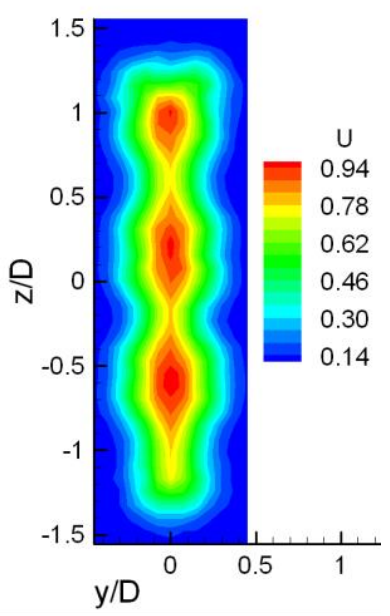

(c)

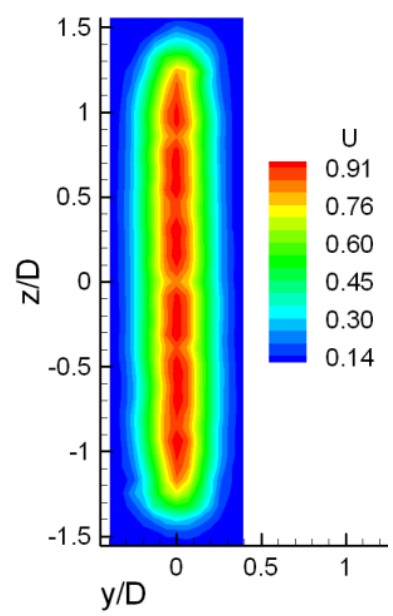


(d)

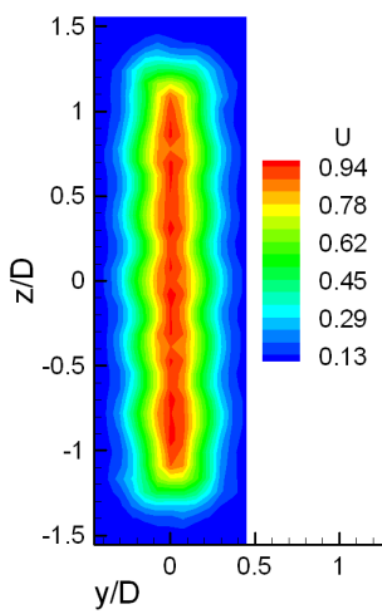

(e)

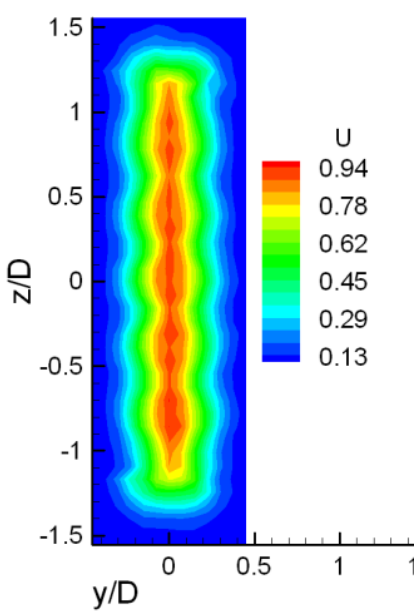

(f)

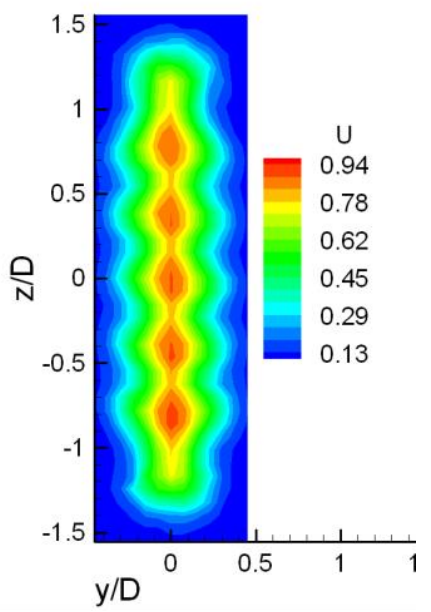

Fig. 4 Mean velocity contours at $x / D=2, M_{j}=0.265$ (single hot-wire data). Cases shown are: (a) Fsh3, (b) Fsh0, (c) 6Rec, (d) Insd, (e) OutF, and (f) Sclp.

(a)

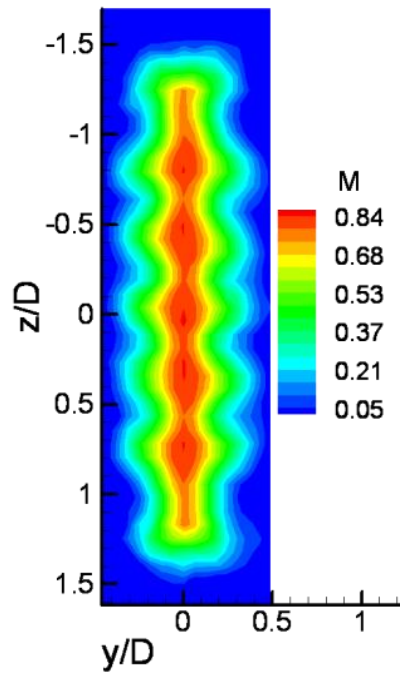

(b)

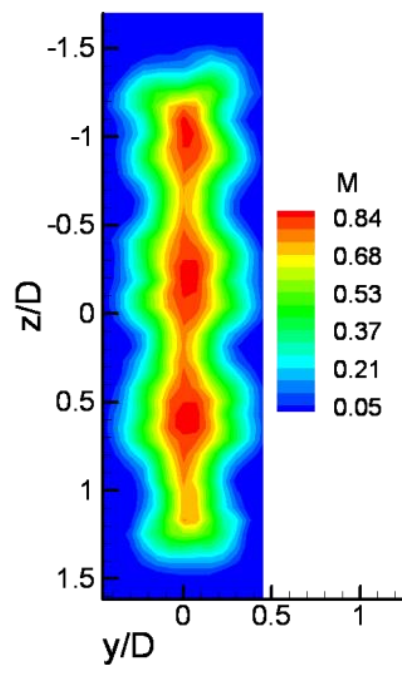

(c)

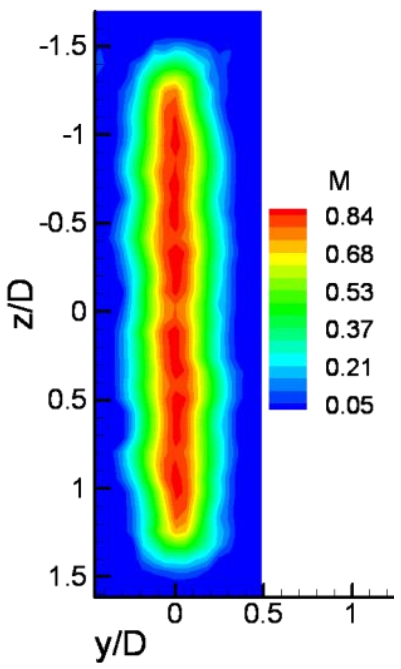

Fig. 5 Mach number contours at $x / D=2, M_{j}=0.90$ (single Pitot probe data). Cases shown are: (a) Fsh3, (b) Fsh0 and (c) 6Rec.
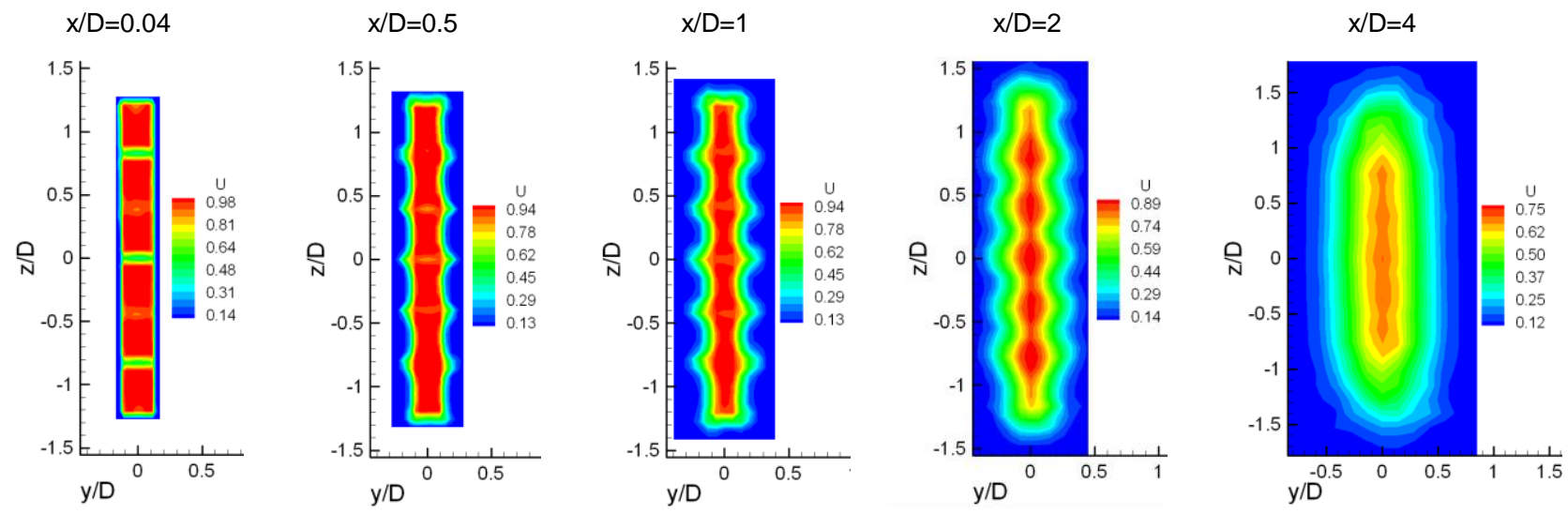
Fig. 6 Evolution of mean velocity field for the Fsh3 case. Data shown for five streamwise $(x / D)$ locations as indicated; $M_{j}=0.265$ (single hot-wire data).
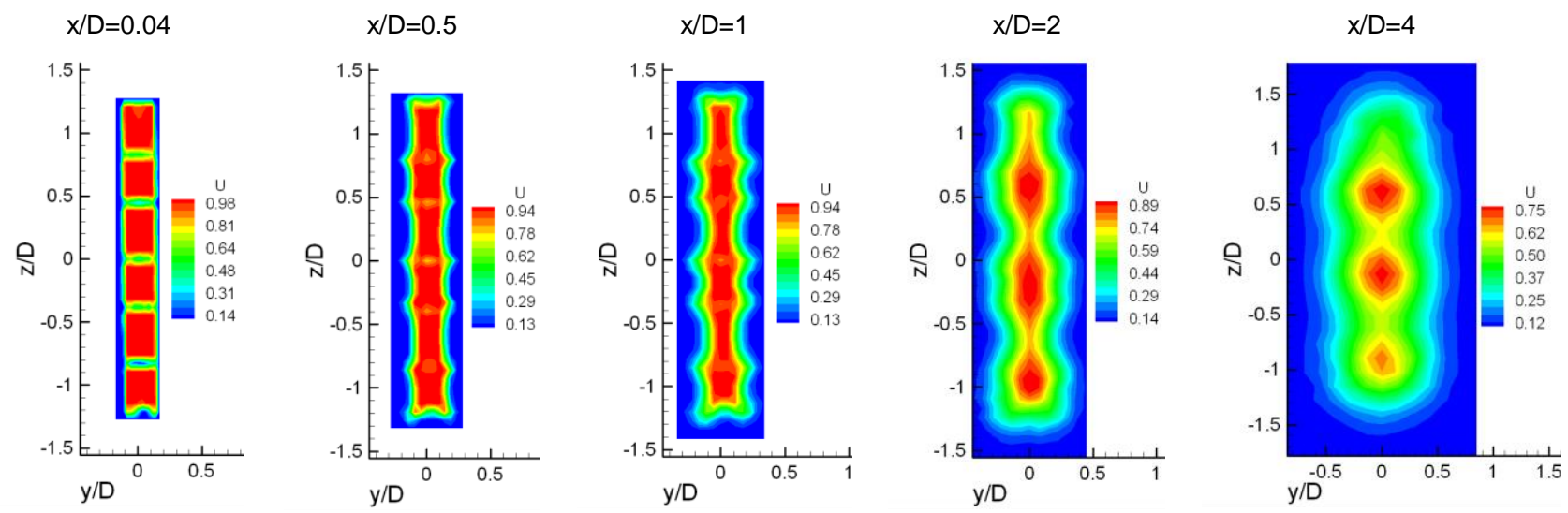

Fig. 7 Evolution of mean velocity field for Fsh0 case. Data shown similarly as in Fig. 6.
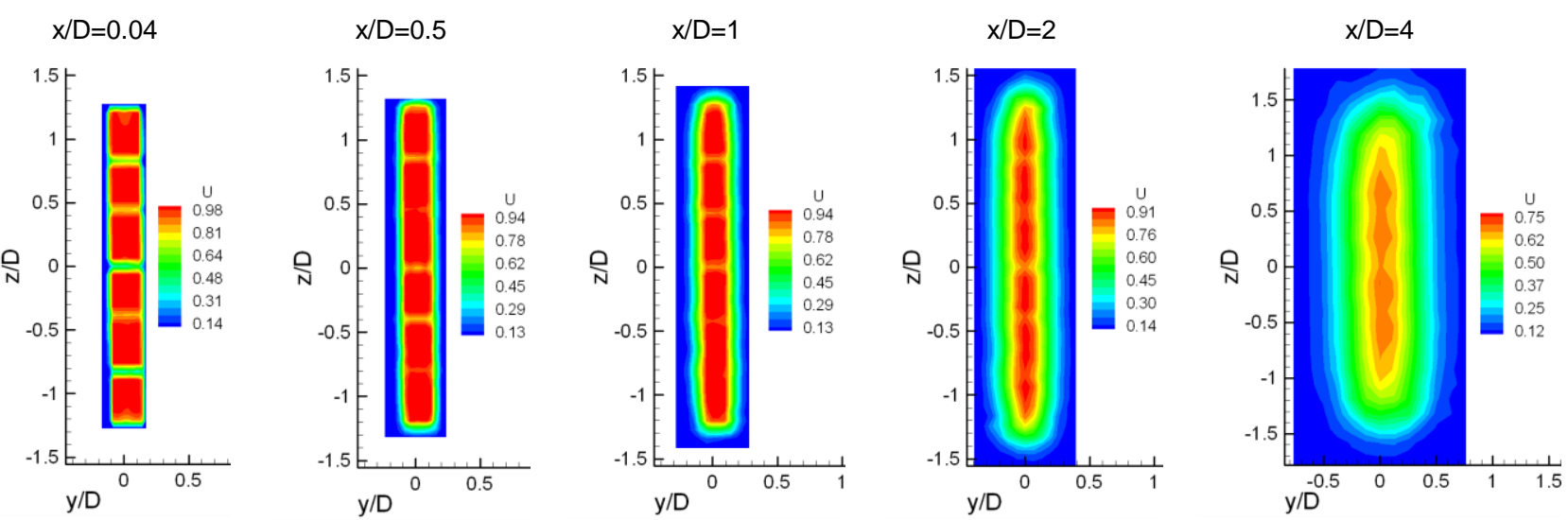

Fig. 8 Evolution of mean velocity field for 6Rec case. Data shown similarly as in Fig. 6. 

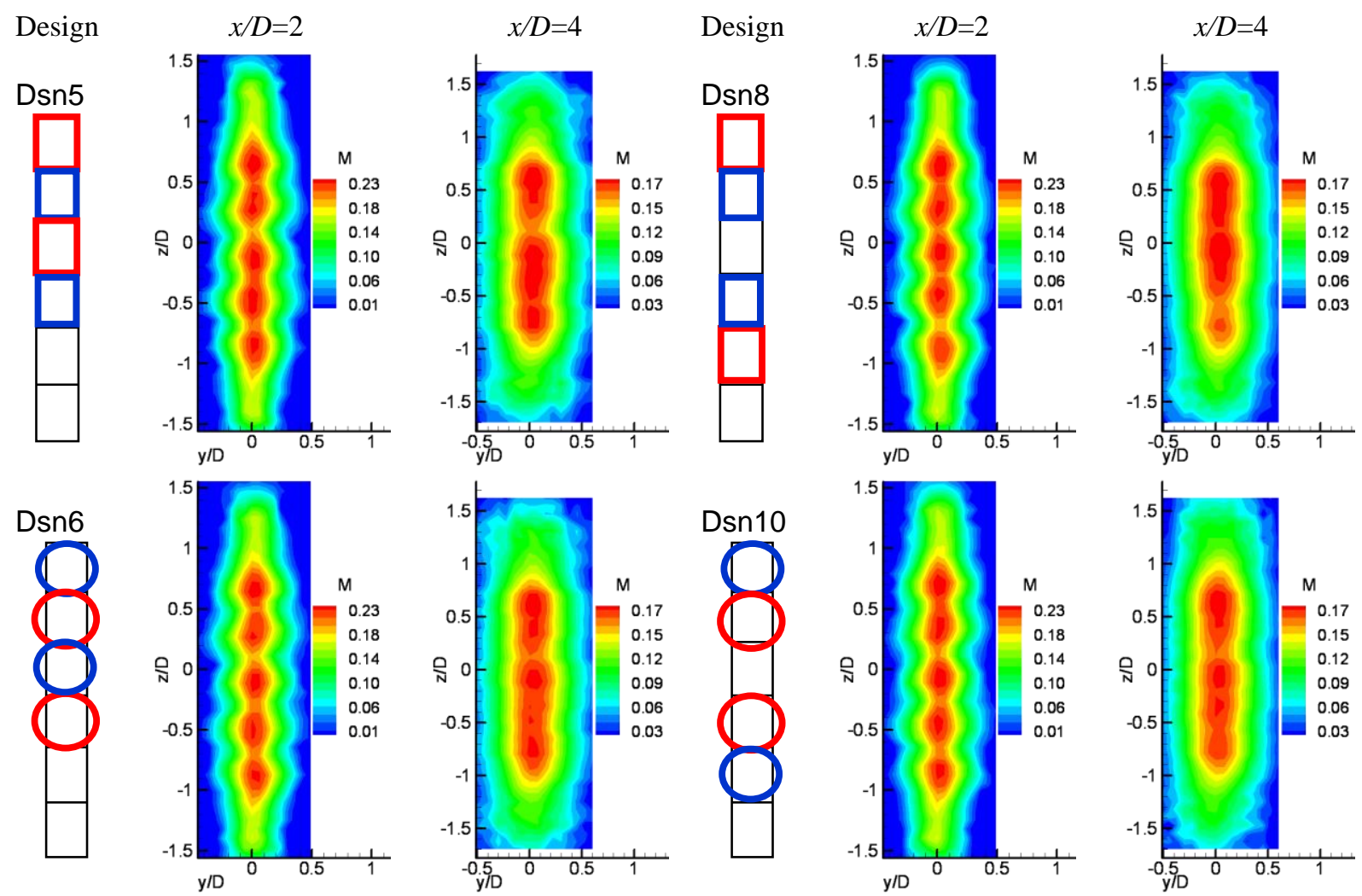

Fig. 9 Mach number distributions at $x / D=2$ and 4 for four designs of the septa passages; design indicated on left side of each case (see text); $M_{j}=0.27$ (Pitot probe rake data).
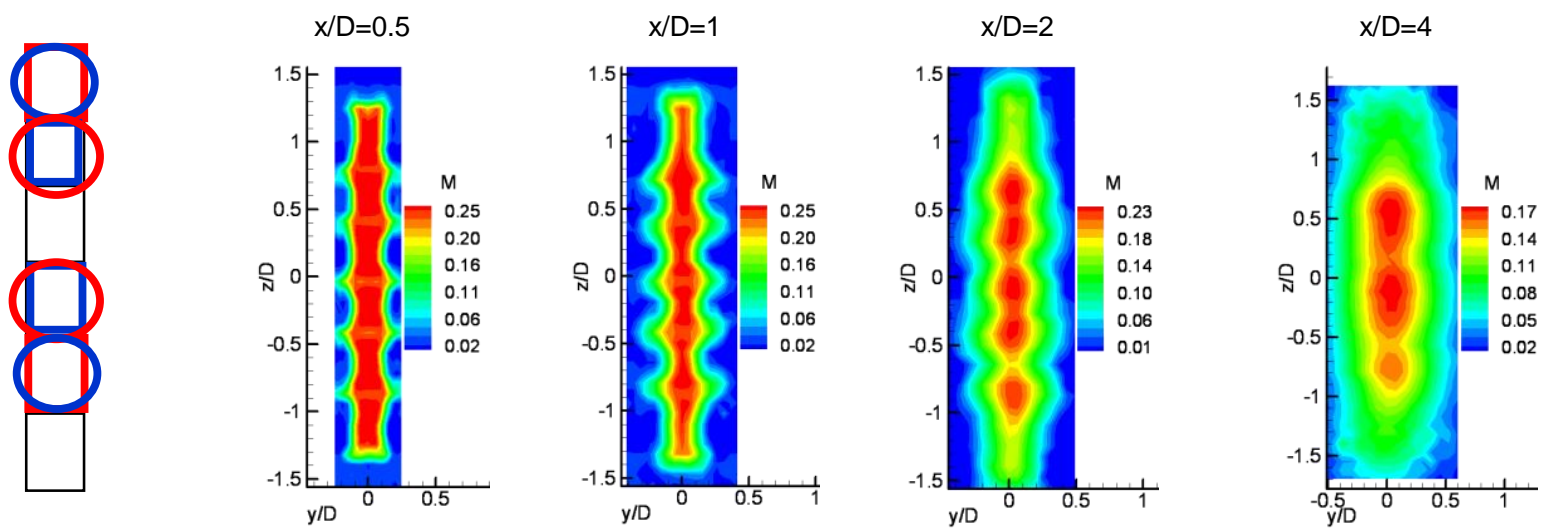

Fig. 10 Evolution of mean velocity (Mach number) field for Dsn9 case; design indicated on left. Data shown for four streamwise $(x / D)$ locations as indicated; $M_{j}=0.27$ (Pitot probe rake data). 

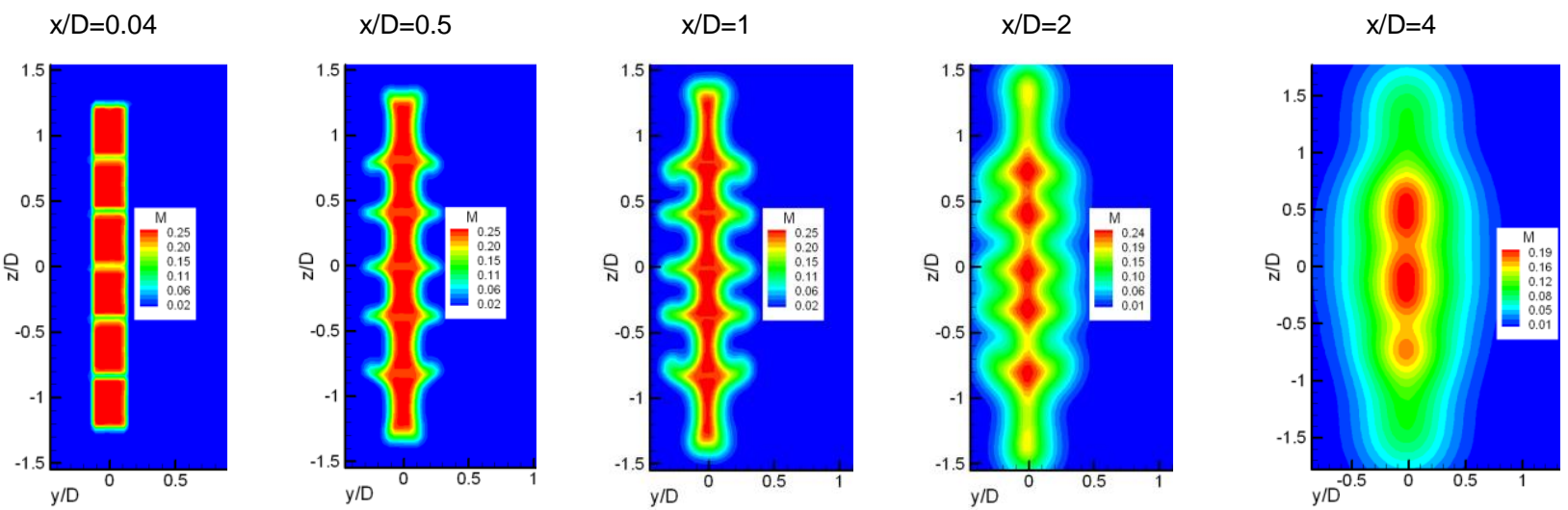

Fig. 11 CFD solution for mean velocity (Mach number) field for Dsn9 case; data shown for five streamwise $(x / D)$ locations as indicated, $M_{j}=0.27$ (compare with Fig. 10).
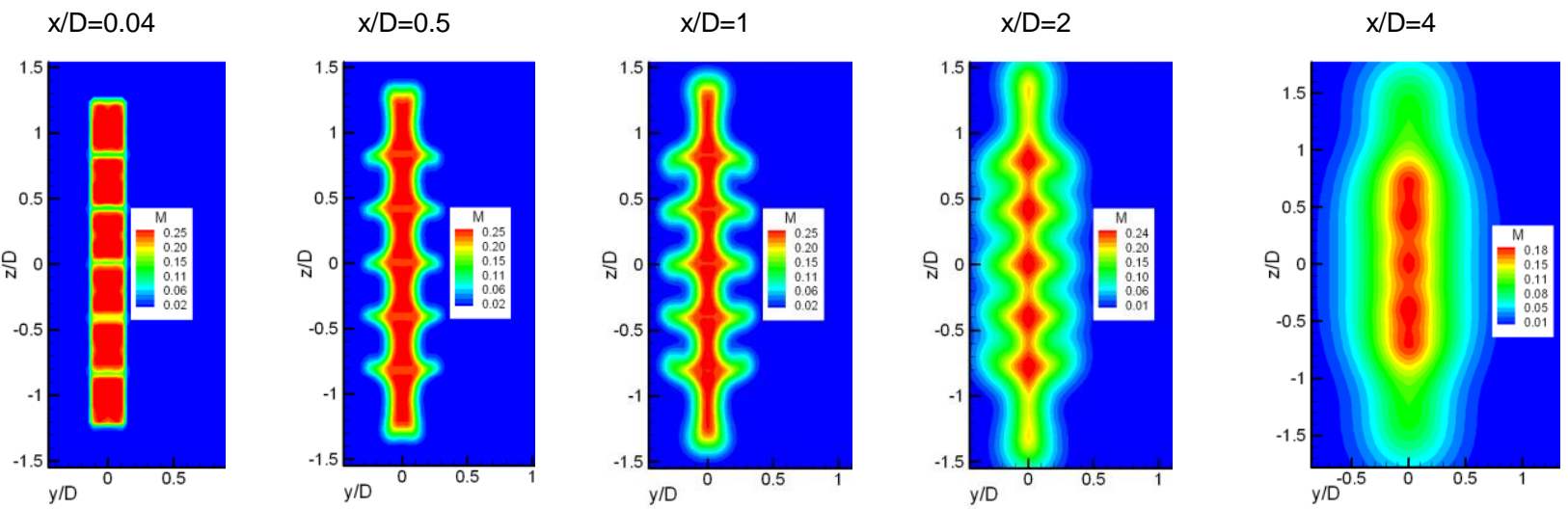

Fig. 12 CFD solution for mean velocity (Mach number) field for Fsh3 case; data shown for five streamwise $(x / D)$ locations as indicated, $M_{j}=0.27$ (compare with Fig. 6).
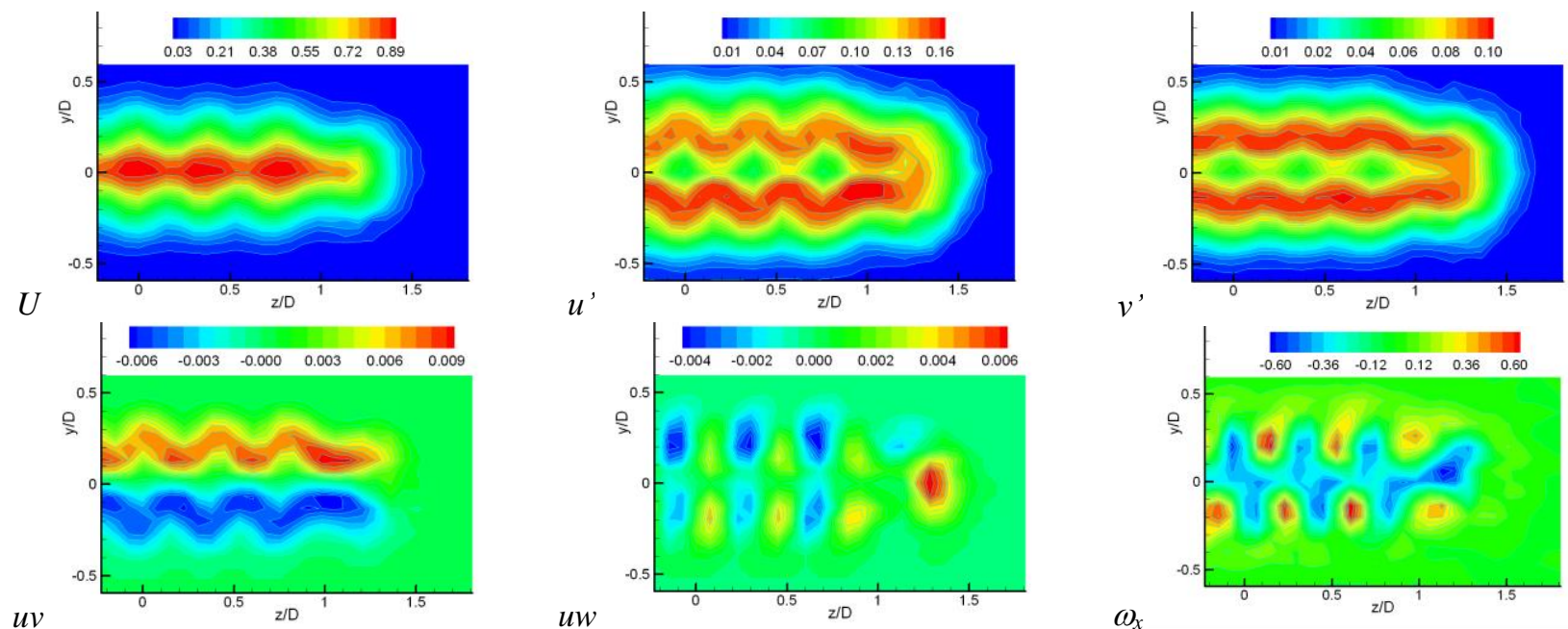

Fig. 13 Distribution of various flow properties for the Fsh3 case at $x / D=2$ (two $\mathrm{X}$-wire measurements). 